\title{
Estimation of Mass Ratio of the Total Kernels within a Sample of In-Shell Peanuts Using RF Impedance Method
}

\author{
Chari V. Kandala and Jaya Sundaram \\ USDA, Agricultural Research Service, National Peanut Research Laboratory, P.O. Box 509, \\ 1011 Forrester Drive, Dawson, GA 39842, USA \\ Correspondence should be addressed to Jaya Sundaram, jaya.sundaram@ars.usda.gov
}

Received 17 September 2009; Accepted 5 November 2009

Academic Editor: Ping-Feng Pai

Copyright ( 2010 C. V. Kandala and J. Sundaram. This is an open access article distributed under the Creative Commons Attribution License, which permits unrestricted use, distribution, and reproduction in any medium, provided the original work is properly cited.

\begin{abstract}
It would be useful to know the total kernel mass within a given mass of peanuts (mass ratio) while the peanuts are bought or being processed. In this work, the possibility of finding the mass ratio while the peanuts were in their shells was investigated. Capacitance, phase angle, and dissipation factor measurements on a parallel-plate capacitor holding in-shell peanut samples were made at frequencies from 1 to $10 \mathrm{MHz}$ insteps of $1 \mathrm{MHz}$. A calibration equation was developed by multilinear regression analysis correlating the percentage ratio of the kernel weight with the measured capacitance, dissipation factor, and phase angle values of in-shell peanut samples with known kernel weights. The equation was used to predict the percentage mass ratio in the validation groups. Fitness of calibration model was verified using standard error of calibration, root mean square error of calibration, and leverage and influence plots. The predictability percentage, within $1 \%$ and $2 \%$ of the visual determination, was calculated by comparing the kernel mass ratio, obtained by the model equation and the reference value obtained by visual determination. Cross-validation gave $96 \%$ and $100 \%$ predictability, and external validation gave $87 \%$ and $98 \%$ predictability within $1 \%$ and $2 \%$ difference, respectively.
\end{abstract}

\section{Introduction}

Peanuts harvested and dried are sold by weight, while they are in their shells. It would be very useful if we can estimate, at the time of buying a load of peanuts, the weight of the kernels we may obtain when this load of peanuts are shelled, without actually shelling the peanuts. Knowledge of the ratio of kernel mass to the total peanut pod mass could be a good indicator of the quality of the peanuts that are being purchased. Up front, it would indicate to the buyer what proportion of the peanuts he is about to buy has useful kernels, and how much is waste. In the traditional way of determining the mass ratio, about $500 \mathrm{~g}$ of peanut sample are shelled and the kernels are separated and weighed. The kernel mass ratio is then calculated as the ratio of the kernel weight to the sample weight. This method is destructive, time and labor intensive. A device which can estimate the mass ratio without shelling the peanuts will be very useful for peanut industry. An attempt was made here, to apply the RF Impedance method for estimating the mass ratio nondestructively. RF Impedance method was successfully used earlier to estimate the moisture content of in-shell peanuts and grains such as wheat and corn [1-6]. In the present work, variation of certain electrical properties of a sample of peanuts at different radio frequencies was used to estimate the relative compactness of the medium. The ratio of the weight of total kernels to the weight of the in-shell peanuts was correlated to the capacitance and phase angle values of a parallel-plate system holding a sample of in-shell peanuts. A calibration equation was developed to predict the total kernel weight and the equation was validated. This method is rapid and nondestructive.

\section{Materials and Methods}

Georgia runner type peanuts harvested in 2008 crop year were used for the total kernel mass ratio estimation. Peanut 
samples were initially cleaned to remove the farm materials. Cleaned peanuts were separated into two groups called calibration and validation groups. Each group had 25 subsample sets. Each subsample set contained about $100 \mathrm{~g}$ to $120 \mathrm{~g}$ of in-shell peanuts. Moisture content of the peanut sample sets was about 7\% (w.b).

2.1. Basic Principles. Certain dielectric related properties of a food material depend on its material composition and density, and the frequency at which they are measured. From earlier studies on shelled, yellow-dent field corn [7], it was found that the dielectric constant increased with moisture content and it was more significant at frequencies $1-20 \mathrm{MHz}$ than at higher frequencies. From the variation of dielectric constant at these frequencies, the MC in single kernels of corn was estimated [8]. The method was extended to single peanut kernels [9] and later to in-shell peanuts [2]. The conduction of RF signals through a medium depends on the density of the medium and some other electrical properties. Assuming similar variations in dielectric constant with the density of the peanut pods (in-shell peanuts), filling the space between the parallel-plates of a capacitor, the mass ratio of peanut kernels was estimated. From the difference of the capacitance values at two frequencies, it should be possible to estimate the density of the food material. But, it was noted that this capacitance difference was a good estimate of the weight of peanut kernels but was highly influenced by the size and shape of the kernels as in the case of moisture estimation [9]. Two other electrical parameters, dissipation factor $D$, and phase angle $\theta$, were also measured at the two frequencies. Two parallel-plates, fixed on the inside walls of a cylindrical tube made of a nonconducting material, form a capacitor system (Figure 1). For high frequency applications, a flat capacitor is desirable because it can achieve lower impedance than a cylindrical capacitor. As the space between these plates is filled with peanut samples, some of the pods filling the cylinder are not in contact with either of the two plates. They are lying in the middle and in contact with the pods around them. Air gaps are generated randomly between the peanuts pods. These air gaps act as additional capacitors, some in series and some in parallel. However, the air gaps remain the same when measurements are taken at the two frequencies, and it was assumed that their contribution to the variation in the values of $C, \theta$, and $D$ at the two frequencies is not significant.

2.2. Equipment. Agilent LCR analyzer was used to transmit and receive RF signals through the in-shell peanuts. Figure 1 shows the experimental set up used to make the RF signal measurements. A cylindrical acrylic tube, fitted with two sets of parallel-plate electrodes, served as the density sensor. The cylinder is $190-\mathrm{mm}$ long, with an internal diameter of 50$\mathrm{mm}$ and has a wall thickness of 7-mm. The electrode assembly for the cylinder consists of two rectangular aluminum plates, 140-mm-long and 50-mm-wide. The electrodes are cemented to the inner walls, as shown in Figure 1, about 25$\mathrm{mm}$ from the ends. The distance of separation between the parallel-plates was $42-\mathrm{mm}$. This cylinder would rest on top of a rectangular acrylic box and in a circular hole, centered

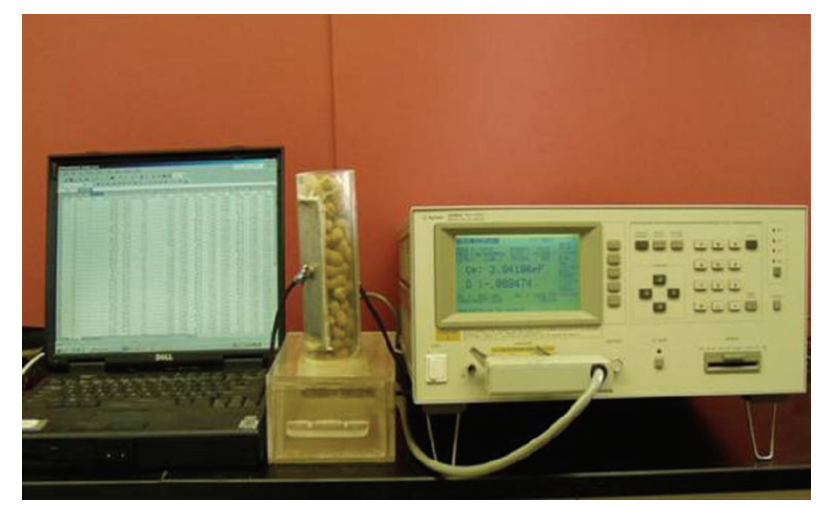

FIgURE 1: Experimental set up of RF signal measuring system.

about 35-mm from the front side of the box. This box is provided with an acrylic drawer that slides in and out of the box manually. The top of this drawer was covered 75-mm from the front end, with an acrylic plate. When the drawer is pushed all the way into the box, this plate covers the hole and prevents the peanut pods placed in the cylinder from dropping into the drawer. Except for the electrodes, no metal parts were used in the assembly of the electrode system or the sample collecting system to prevent any interaction with the RF signal used in the measurements. With the drawer pushed all the way in, the cylinder was filled with peanut pods and the impedance measurements were taken. After the completion of the measurements, the drawer is pulled out slowly, allowing the sample to fall into the drawer. The drawer was emptied before another sample was placed in the cylinder for measurement. With the peanut sample occupying the space between the electrodes, the analyzer measured the impedance and phase angle of this electrode system at $1 \mathrm{MHz}$ to $10 \mathrm{MHz}$, in $1.0 \mathrm{MHz}$ intervals and a computer controlled and collected the data from the analyzer.

2.3. Procedure. Peanut samples from a subsample set were transferred into the cylinder, filling it up to the top of the cylinder and covering the space between the two electrodes (Figure 1), with the drawer fully inserted into the box. The capacitance, dissipation factor, and phase angle were measured for the sample. At the end of the measurements, the sample was emptied in to the drawer from which it was again filled into the cylinder and measurements were taken again. This procedure was repeated ten times for each subsample to account for any variations in the impedance values that may occur, due to the peanut pods orienting differently as they settle in the cylinder, each time it is filled. After 10 measurements the subsample was weighed and separated into shells and kernels. Weights of all the kernels and shells in this subsample were recorded separately. This procedure was repeated for the other 24 sample sets in the calibration group and the 25 samples in the validation group. All the measurements were taken at room temperature of $20-22^{\circ} \mathrm{C}$ and the samples were maintained at that temperature. 
2.4. Data Analysis. NIR spectral data were analyzed using multivariate data analysis software (Unscrambler Version 9.7 CAMO ASA, USA). Using data from the calibration dataset, Multilinear regression (MLR) analysis was conducted to develop an empirical equation to estimate the peanut kernel mass ratio. Though measurements of capacitance, phase angle, and dissipation factor were taken at ten frequencies between $1 \mathrm{MHz}$ and $10 \mathrm{MHz}$, MLR analysis on the data generated with three frequencies $1 \mathrm{MHz}, 5 \mathrm{MHz}$, and $9 \mathrm{MHz}$ was found to be adequate for the prediction. The performance of the calibration equation was verified based on Standard Error of Calibration $(\mathrm{SEC})\left(\mathrm{SEC}=\left((1 /(n-p-1)) \sum_{i=1}^{n} e_{i}^{2}\right)^{1 / 2}\right.$, where $n$ is the number of observations, $p$ is the number of variables in the regression equation with which the calibration is performed, and $e_{i}$ is the difference between the observed and reference value for the $i$ th observation) and coefficient of multiple determinations $\left(R^{2}\right)$. The MLR calibration equation was then used to predict the kernel mass ratio from the measured data of the validation group. Goodness of fit was evaluated based on the Standard Error of Prediction (SEP) $\left(\mathrm{SEP}=\left((1 /(n-1)) \sum_{i=1}^{n}\left(e_{i}-\bar{e}\right)^{2}\right)^{1 / 2}\right.$, where $n$ is the number of observations, $e_{i}$ is the difference in the moisture content predicted and that determined by the reference method for the $I$ th sample, and $\bar{e}$ is the mean of $e_{i}$ for all of the samples) obtained by comparing the reference values with the predicted values. Leverage and influence plots were also used to verify the fitness of the model. Reference values were obtained by the traditional shelling, separating, and weighing method.

\section{Results and Discussion}

From the measured values of capacitance, phase angle, and dissipation factor at frequencies $1 \mathrm{MHz}, 5 \mathrm{MHz}$, and $9 \mathrm{MHz}$ and the reference mass ratio value obtained by traditional methods on the samples in the calibration lot, the calibration equation developed to predict the mass ratio was as below:

$$
\begin{aligned}
\% \text { Mass Ratio }= & -0.00176-38.77 C_{1}+27.03 C_{5} \\
& +27.20 C_{9}+19.10 \theta_{1}-8.92 \theta_{5}+11.28 \theta_{9} \\
& +638.38 D_{1}-205.27 D_{5}+776.43 D_{9},
\end{aligned}
$$

where, $C_{1}, \theta_{1}, D_{1} ; C_{2}, \theta_{2}, D_{2}$, and $C_{3}, \theta_{3}, D_{3}$ are the capacitance, phase angle, and dissipation factors at $1 \mathrm{MHz}, 5 \mathrm{MHz}$, and $9 \mathrm{MHz}$, respectively.

Shown in Table 1 are the fitness measures of MLR calibration model developed using in-shell peanuts from the calibration group. Though the coefficient of determination has a slightly low value of 0.55 , a low value of 0.53 for the SEC and a very low bias value indicate the usefulness of the model for the mass ratio prediction.

In Table 2 the fitness measures of the model equation when used to predict the kernel mass ratio of the validation group of in-shell peanuts are shown. The SEP and RMSEP values for the prediction are considerably less than 1.0. The bias value is also quite low.
TABLE 1: Fitness measures of MLR model on calibration group of in-shell peanuts.

\begin{tabular}{lc}
\hline Fitness parameter & Value \\
\hline Standard error of calibration (SEC) & 0.53 \\
Root mean square error of calibration (RMSEC) & 0.52 \\
Bias & $-6.6 \times 10^{-5}$ \\
Coefficient of determination $\left(R^{2}\right)$ & 0.55 \\
\hline
\end{tabular}

TABLE 2: Fitness measures of MLR model on validation group of in-shell peanuts.

\begin{tabular}{lc}
\hline Fitness parameters & Value \\
\hline Standard error of prediction (SEP) & 0.81 \\
Root mean square error of prediction (RMSEP) & 0.81 \\
Bias & -0.02 \\
\hline
\end{tabular}

TABLE 3: Total kernel ratio predicted by the model.

\begin{tabular}{lcc}
\hline \multirow{2}{*}{ Peanut groups } & \% prediction within a difference* of \\
& $1 \%$ & $2 \%$ \\
\hline Calibration group & 100 & 100 \\
Cross-validation & 96 & 100 \\
Validation group & 87 & 98 \\
\hline
\end{tabular}

* Total kernel mass ratio predicted within $1 \%$ and $2 \%$ difference of the mass ratio obtained using traditional visual method.

Shown in Table 3 is the percentage of mass ratio of total kernel predictability obtained using the MLR model equation. The predictability percentage was calculated by comparing the kernel mass ratio values predicted, using the model equation, and the reference values obtained using traditional shelling and weighing method. Predicted values were calculated for both calibration and validation groups using (1). It can be seen that the mass ratio could be predicted for $100 \%$ of the samples in the calibration group within $1 \%$ of the values determined by the traditional method. When the model equation was used for cross validation, it gave $96 \%$ predictability within $1 \%$ difference, and for prediction using validation group peanuts, not used in the calibration, it gave $87 \%$ predictability within $1 \%$. The predictability was better than $98 \%$ in all cases when considered within $2 \%$.

Figure 2 shows the probability plot of $Y$ residuals. $Y$ variable represented the mass ratio of kernel to the total inshell peanuts. This plot shows the cumulative distribution of the $Y$ residuals representing the variation in kernel mass ratio. It is normally distributed and appears along a straight line. It shows that most of the residuals are along the straight line. This indicates that the calibration model was developed by taking into account all variations in the $Y$ variables. However, it can be seen in the plot that, there are some deviations of the residuals from the straight line. The deviations were not considered as too prominent and those residuals were not considered as true outliers.

Leverage plot, shown in Figure 3, is the distance between the object projections and the model center, which is the origin around the variations in the data that was used to 


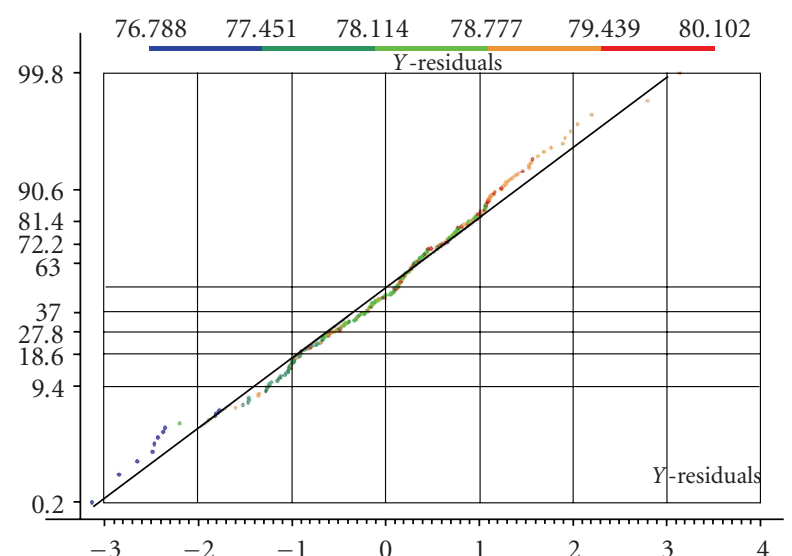

FIgURE 2: $Y$-residuals normal probability plot.

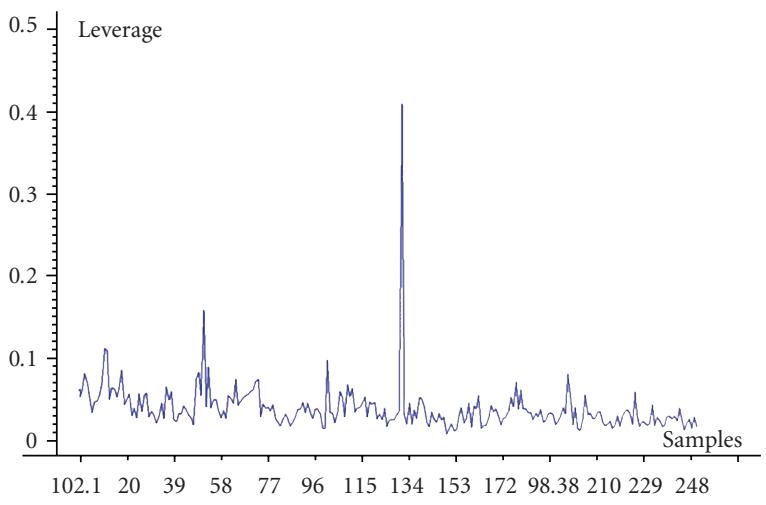

Figure 3: Sample leverage plot for calibration model.

develop the calibration model. Leverage plot identifies any sample that is far away from the model center. If it is an outlier it is not well represented by the developed model. Higher the leverage value is, higher its influence on the model is. Leverage is indicated in absolute values which vary from $0-1$. As a rule of thumb, a sample with a numerical value of leverage over 0.4 or 0.5 can adversely affect the model. It can be seen from Figure 3 that no sample point has a high leverage value except sample 131, which has a leverage value of 0.4 that is marginally high. This one point is not expected to distort the model.

Figure 4 displays the difference between the reference mass ratio and predicted mass ratio for each sample. These differences were plotted against sample leverages. This type of plot is useful in identifying the dangerous outliers. In this plot, samples with high residual variance that is, lying at the top of the plot might be the outliers or they could be the samples not well represented by the calibration model. Samples with high leverage, that is, lying at the right side of the plot are called influential. These are the samples not dangerous as they are described well by the $X$ values in the model. Sample 131 in Figure 4 is thus an influential sample. Samples with high leverage and residual $Y$-variance are only considered as dangerous outliers. From Figure 4, it could be seen that no sample had fallen in the dangerous zone. Thus, it

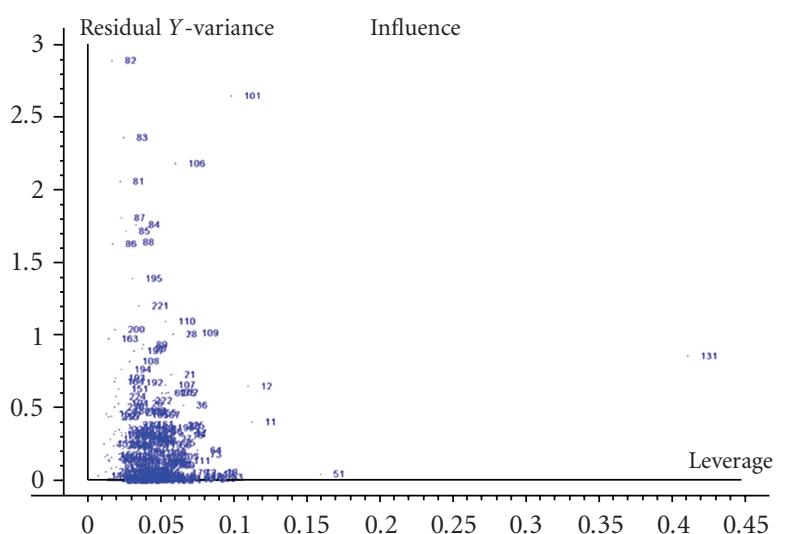

FIgURE 4: Residual $Y$-variance influence plot for calibration model.

can be concluded that the calibration MLR model developed to predict the mass ratio is quite adequate.

\section{Conclusions}

This study showed that by measuring capacitance, phase angle, and dissipation factor of a capacitance system, holding in-shell peanuts between them, a good estimate of total kernel mass ratio relative to the original peanut mass could be obtained. Influence plot made using $Y$-variance residuals and leverage plot showed that very few of the samples measured qualified to be designated as outliers. Also, they were seen to be nondangerous outliers that do not affect the calibration model developed using MLR. It was also evident from the $Y$-residual probability plot that the outliers were not true outliers. Predictability percentage obtained by comparing MLR model and visual determination within a difference of $1 \%$ was $87 \%$ for external validation and $96 \%$ for cross validation. These results showed that RF impedance measurements are useful for the rapid and nondestructive estimation of total kernel mass ratio in a given sample of in-shell peanuts. This method would be useful to get an initial estimate of the kernel mass ratio without shelling the peanuts.

\section{References}

[1] C. V. K. Kandala and S. O. Nelson, "Nondestructive moisture determination in small samples of peanuts by RF impedance measurement," Transactions of the American Society of Agricultural and Biological Engineers, vol. 48, no. 2, pp. 715-718, 2005.

[2] C. V. K. Kandala and S. O. Nelson, "RF impedance method for nondestructive moisture content determination for in-shell peanuts," Measurement Science and Technology, vol. 18, no. 4, pp. 991-996, 2007.

[3] C. V. Kandala and C. L. Butts, "Phase angle and impedance measurements for nondestructive moisture content determination of in-shell peanuts using a cylindrical sample holder," in Proceedings of the ASABE Annual International Meeting, vol. 13, Minneapolis, Minn, USA, June 2007, 07-6215.

[4] S. O. Nelson, "RF and microwave dielectric properties of shelled, yellow dent field corn," Transaction of the American Society of Agricultural Engineers, vol. 22, no. 6, pp. 1451-1457, 1979. 
[5] T. Ban and M. Suzuki, "Studies on electrical detection of grain moisture in artificial drying," Tech. Rep. 11, Institute of Agricultural Machinery, Omiya, Japan, 1977.

[6] S. O. Nelson, "Use of electrical properties for grain moisture measurement," Journal of Microwave Power, vol. 12, no. 1, pp. 67-72, 1977.

[7] S. O. Nelson, "Frequency and moisture dependence of the dielectric properties of high-moisture corn," Journal of Microwave Power, vol. 13, no. 2, pp. 213-218, 1978.

[8] C. V. K. Kandala, S. O. Nelson, and K. C. Lawrence, "Nondestructive electrical measurement of moisture content in single kernels of corn," Journal of Agricultural Engineering Research, vol. 44, pp. 125-132, 1989.

[9] C. V. K. Kandala and S. O. Nelson, "Measurement of moisture content in single kernels of peanuts: a nondestructive electrical method," Transactions of the American Society of Agricultural Engineers, vol. 33, no. 2, pp. 567-571, 1990. 

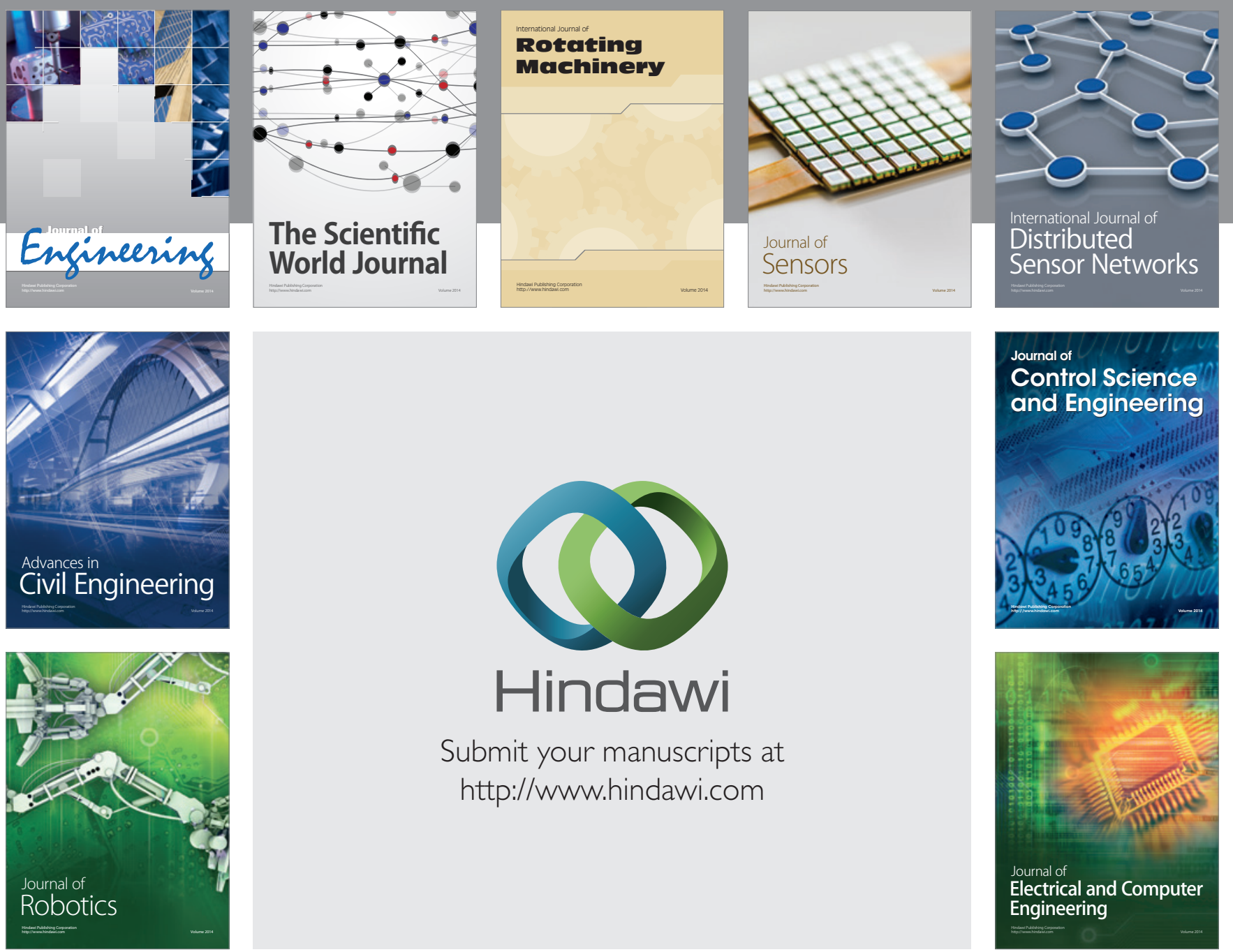

Submit your manuscripts at

http://www.hindawi.com
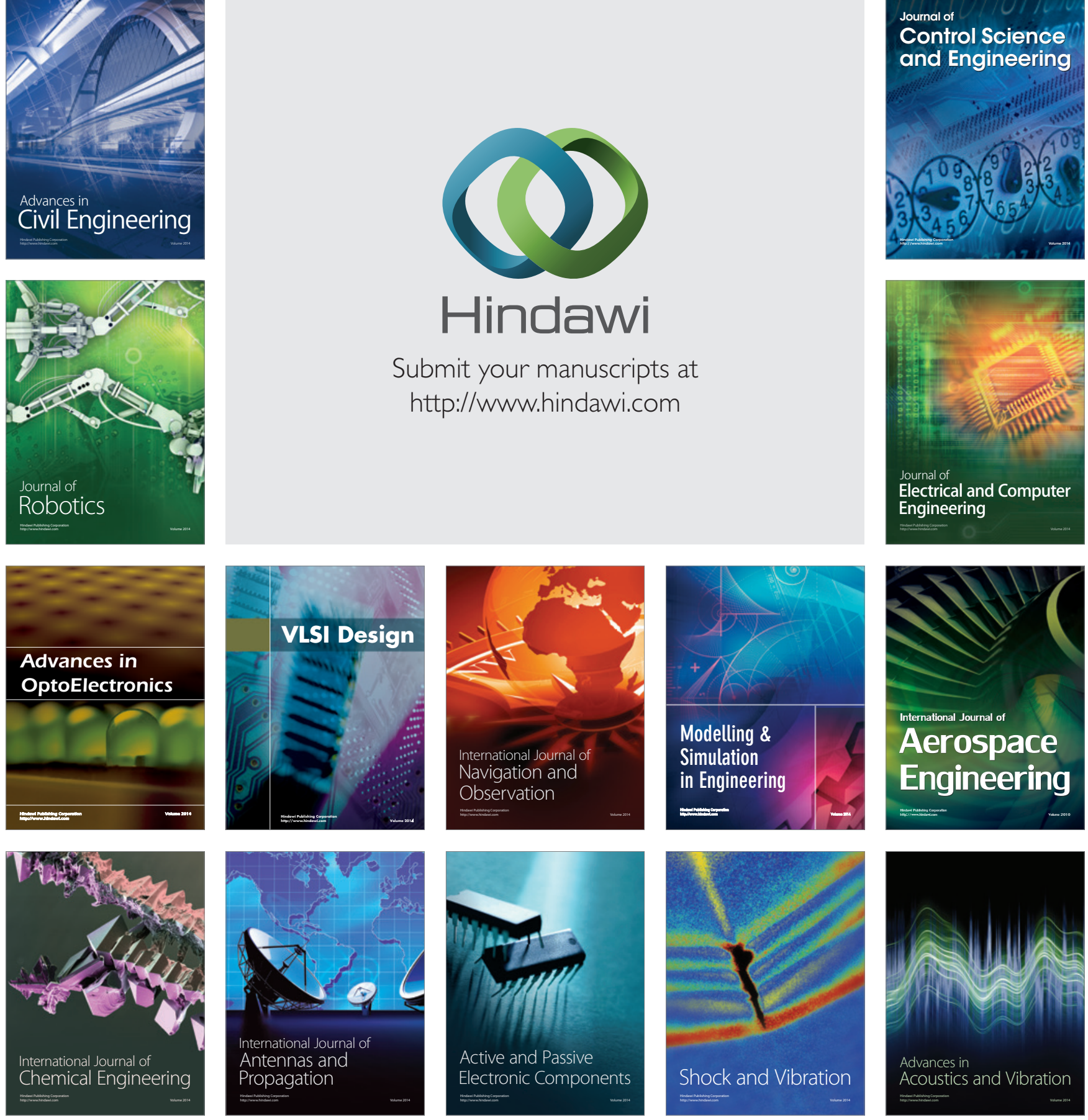